\title{
ESTADO DEL ARTE EN REDES VANET Y CADENAS DE MARKOV
}

\section{A SURVEY ON VANET NETWORKS AND MARKOV CHAINS}

\begin{abstract}
This article summarizes the state-of-the-art VANET networks as an extension of MANET networks, which have particular characteristics in terms of how to build topologies that change dynamically over time; in that sense, we propose that, within the arguments of future research, analysis should stem not from a simulation point of view but from a mathematical point of view to be subsequently developed through Markov chains. This is plausible since the formation of VANET networks corresponds to, or can be viewed as, a discrete stochastic process, which can be mathematically analyzed by means of these chains in order to ensure reliable delivery of information packets between vehicles that are constantly coming and going within the network topology.
\end{abstract}

Keywords: MANET, VANET, markov chains, protocols, algorithms, standards.

\section{Resumen}

El presente artículo resume el estado del arte de las redes VANET como una extensión de las redes MANET, por tener características particulares en cuanto a la forma de construir topologías que cambian dinámicamente en el tiempo, en tal sentido, se proponen los argumento para que en futuros trabajos de investigación, se haga un análisis no desde el punto vista de simulación, sino desde el punto de vista matemático para que se desarrolle a través de cadenas de Markov, ya que la construcción de redes VANET corresponde o puede verse como un proceso estocástico discreto, que puede ser analizado matemáticamente por medio de dichas cadenas, con el fin de garantizar una entrega confiable de paquetes de información entre vehículos los cuales están entrando y saliendo constantemente dentro de la topología de red.

Palabras clave: MANET, VANET, cadenas de Markov, protocolos, algoritmos, estándares.
Jorge I. Blanco

Ingeniero Electrónico. Estudiante de la maestria en Ciencias de la Información y las Comunicaciones de la Universidad Distrital Francisco José de Caldas. Bogotá, Colombia.

jorgeblanco@gmail.com

Cesar A. Hernandez Suarez Ingeniero Electrónico, Especialista en Redes, Magister en Ciencias de la Información y las Comunicaciones, candidato a Doctor en Ingeniería de Sistemas y Computación. Docente de la Universidad Distrital Francisco José de caldas. Bogotá, Colombia.

cahernandezs@udistrital.edu. co

Octavio J. Salcedo Parra

Ingeniero de Sistemas, Magister en Teleinformática de la Universidad Distrital, magister en Economía, se desempeña como docente de planta de la Universidad Distrital "Francisco José de Caldas"

ojsalcedop@udistrital.edu.co

Tipo: Artículo de revisión

Fecha de Recepción: Oct. 4 de 2010

Fecha de Aceptación: Nov. 5 de 2010 


\section{INTRODUCCIÓN}

En la última década, las redes vehiculares han empezado a despertar un gran interés tanto a nivel de universidades como en la industria automotriz, a través de sus centros de investigación, al igual que operadores de redes como: Orange Lab, AT\&T, Telecom Italia, de igual forma, las autoridades gubernamentales se encuentran a la expectativa del desarrollo y evolución de esta tecnología a fin de apoyar con la legislación correspondiente; esto ha generado un tema de estudio y análisis riguroso y permanente por parte de los diferentes actores, llevando ya a la definición de estándares a través de organizaciones como la IEEE, la FCC, la Comunidad Europea, etc., por su parte, países como Japón, Taiwán, Malasia, Estados Unidos, la Unión Europea, etc., son entre otros, quienes están liderando sobre esta iniciativa, reflejado en significativos avances y contribuciones.

El estudio de las redes vehiculares de próxima generación corresponde a una tecnología que apenas, podría decirse, está en fase de investigación, lo cual muestra la gran cantidad de oportunidades que van desde el desarrollo de aplicaciones, dispositivos, protocolos, estándares, regulaciones, modelos de negocio, prestación de servicios, infraestructuras celular, integración de tecnologías, calidad de servicio, seguridad, encaminamiento, redes satelitales, acceso a información y entretenimiento a través de Internet (Infotainment) ${ }^{1}$. Esto despierta una gran motivación para profundizar en todos los aspectos que involucran las redes VANET, más aún, mirando quienes también están liderando estas iniciativas encontramos universidades como el MIT, compañías como Cisco, Google ${ }^{2}$, Mi-

1. http://jamillan.com/v_infot.htm

2.http://www.technologyreview.com / communications/25340/?mo crosoft ${ }^{3}$, fabricantes como Toyota, Renault, etc., por lo cual, muestra no solo las expectativas alrededor de éste tema sino la seriedad y el compromiso que hay frente a esta clase de redes, dados los actores involucrados, por lo tanto, desde éste artículo, cuyo tema de redes VANET, corresponde a un trabajo de maestría, se invita a la universidad colombiana y dada su misión, a unirse a estas tendencias mundiales, en razón a la importancia e impacto que puede tener en la sociedad, en especial, cuando se ha revisado la literatura y no se ha encontrado a la fecha que alguna institución universitaria de Colombia ni de países de la región, a excepción de pocos casos de estudio en México y España, por su parte, lo más próximo y parecido para el caso colombiano es el lanzamiento para el 2012 del Centro de Desarrollo de la Industria Automotriz, el cual "corresponde a una de las iniciativas del plan de negocios de la industria de autopartes y vehículos, con el que se busca enfocar la gestión del conocimiento a través de redes de investigación, desarrollo e innovación, de forma tal que le permita a la industria automotriz colombiana avanzar para convertirse en un sector de talla mundial" 4 . En tal sentido, se espera que las redes VANET, formen parte de la agenda de investigación de dicho centro de desarrollo automotriz.

\section{CONCEPTOS DE REDES VANET}

VANET (Vehicular Ad-hoc Network) [1] En términos generales las redes VANET se definen como una clase de red inalámbrica [2] derivada de las redes MANET (Mobil Ad-hoc Networks), que han surgido gracias a los avances tanto en las tecnologías inalámbricas e investigaciones en la industria automotriz para desarrollar redes que forman topologías espontá-

3.http: / / www.technologyreview.com / communications $/ 37552 / ? \mathrm{a}=\mathrm{f}$

4. http://claris1234.blog.com.es/ 
neas entre vehículos en movimiento a diferentes velocidades, equipados con interfaces inalámbricas que pueden ser con tecnologías homogéneas o heterogéneas permitiendo comunicaciones entre vehículos cercanos o equipos con infraestructuras a fin de transmitir o compartir diferentes tipos de información [3]. En las redes vehiculares, cada vehículo es equipado con la tecnología necesaria para permitir capturar información de sí mismo como de su entorno, esta información no solo debe ser procesada para la toma de decisiones del mismo vehículo sino que también para ser transmitida a los demás vehículos adyacentes o dentro de la topología. La fig. 1 [3]. muestra un ejemplo de red vehicular en el cual se visualizan los tres tipos de comunicaciones que pueden existir para el intercambio de mensajes, estos son: comunicación intra-vehicular, de vehículo a vehículo y de vehículo a infraestructura.

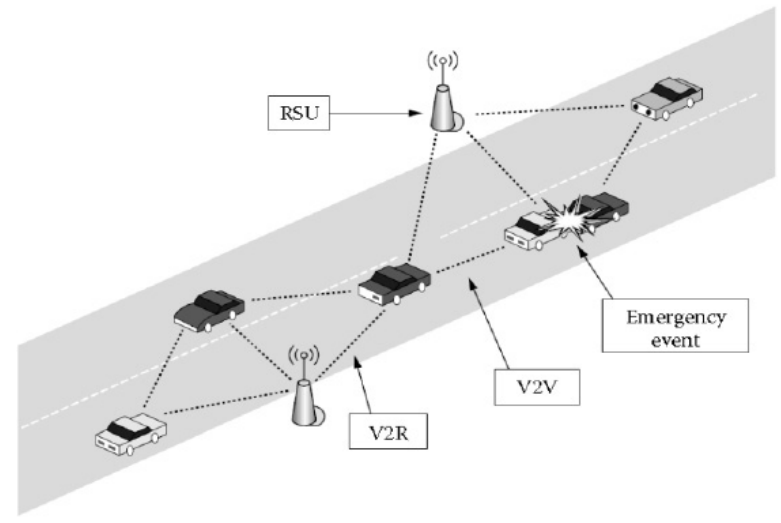

Fig. 1. Ejemplo de una red vehicular.

El entorno intra-vehicular se lleva a cabo a través desensores y dispositivos electrónicos como actuadores[4] que procesan la información capturada por los sensores, para luego ser procesada y tomar las acciones pertinentes, la comunicación se da a nivel del mismo vehículo y los dispositivos que realizan estas tareas se conocen como OBUs (On Board Units) o Unidades a bordo; por su parte, la comunicación entre vehículos (V2V) se hace también a través de enlaces inalámbricos y se basan en el concepto de comunicaciones Ad-hoc [5] en el cual se presenta una topología cambiante de forma dinámica por tratarse de nodos que están en movimiento y a su vez dispersos a lo largo de la vía; el otro tipo de comunicación que puede darse a nivel de las redes vehiculares es de vehículo a infraestructura (V2I), llamada generalmente RSU (Roudside Unit) por sus siglas en inglés, las RSU, son el equivalente a los puntos de acceso en las redes inalámbricas convencionales $\mathrm{y}$ su propósito es ayudar en el suministro y recolección de información para los vehículos también conocidos como nodos móviles, básicamente es un mecanismo de respaldo a las comunicaciones vehículo a vehículo (C2C).

Las redes VANET [6] son donde más se centra el foco de atención e investigación actualmente, ya que como se ha dicho, son redes impredecibles, su topología permanentemente está cambiando en tiempo y posición, presentan fragmentación temporal de la red, son muchos los factores y variables involucrados, los cuales las hacen algo diferentes con respecto a las MANET, pero aun así, es en este tipo de redes donde está la esperanza de desarrollar Sistemas de Transporte Inteligente (ITS) [7], por su sigla del inglés, por esto mismo, ya está ratificado el estándar IEEE 802.11p (DSRC, Dedicated Short Range [8] Communication) ${ }^{5}$, que busca regular el envío de información en entornos vehiculares donde emplea una serie de mecanismos de diferenciación de servicios mediante la creación de interfaces que permiten administrar el servicio de los paquetes según prioridad establecida, 802.11p [9] tiene asignada la banda de $5.9 \mathrm{GHz}$ [10], con un rango de frecuencias de $75 \mathrm{MHz}$ para Estados Unidos y $30 \mathrm{MHz}$ para Europa.

Las redes VANET son un subconjunto

5. http://en.wikipedia.org/wiki/Dedicated_Short_ Range_Communi 
de las llamadas MANET (Mobile Ad Hoc Networks [11]). Las redes MANET pertenecen al grupo de trabajo en el área de enrutamiento de la IETF (Internet Engineering Task Force), que es la organización que define los diferentes RFCs (Request For Comments). Haciendo una búsqueda en el sitio Web de la IETF, se encuentra que hay alrededor de unos 30 documentos entre RFCs y Active Internet-Drafts, relacionados con las redes MANET $^{6}$ [12]

Existen varias formas de clasificar los protocolos de encaminamiento[13-15]:

- De acuerdo al alcance:

Unicast. Transmiten información de un único emisor a un único receptor.

Broadcast. Se envía la información a todos los nodos de una red.

Anycast. El destinatario es único pero uno cualquiera no especificado.

Multicast. La información se envía a un grupo de nodos.

Geocast. Un caso especial del multicast para las redes VANET[16], en el cual se define el envío de información desde un emisor a un grupo de receptores situados en cierta área geográfica, donde no es necesario unirse a un grupo previamente establecido; solo por su posición geográfica un nodo recibirá o no los datos transmitidos.

- Según al esquema de descubrimiento de rutas de acuerdo al algoritmo:

Proactivos. Periódicamente actualizan las rutas, tienen conocimiento de la topología de la red en todo momento intentando tener optimizadas las rutas o Reactivos. Buscan la ruta hacia un destino en el momento en el que necesitan enviar información.

Híbridos. Combinan los dos esquemas, proactividad en las cercanías del nodo y

6. http://datatracker.ietf.org/wg/manet/ reactivos tratando de buscar las rutas a nodos alejados cuando es requerido.

- De acuerdo al tipo de algoritmo en que se fundamentan [17]:

Vector de distancias. Los nodos en una red intercambian periódicamente sus vectores de distancia con sus vecinos, de tal manera que un nodo recibe de sus estos sus respectivos vector-distancia, con esa información y sabiendo la distancia estimada a cada uno de sus vecinos, un nodo puede decidir por cuál de estos se consigue la mejor ruta para llegar a un destino. Estado enlace. Los nodos monitorizan el estado de los enlaces con sus vecinos y difunden esta información; cada nodo conoce el estado de todos los enlaces y toda la topología; con esta información puede calcular las mejores rutas a cada destino.

Basados en información geográfica. Se hacen cálculos de dónde se estima que pueda estar el o los nodos destinos y se encamina la información en esa dirección.

Basados en zonas. Dividen la red en subzonas y dependiendo de en qué zona se encuentre un nodo, puede con la información recibida descartarla, reenviarla o enviarla de acuerdo al alcance unicast, multicast, anycast, broadcast o geocast.

A continuación se relacionan algunos de los protocolos más estudiados por diferentes comunidades de investigación, teniendo en cuenta la clasificación descrita en el párrafo anterior [18]:

- FSR (Fisheye state routing protocol. Unicast proactivo).

- DSR. (Dynamic source routing protocol. Unicast reactivo).

- AODV. (Ad hoc on demand distance vector. Unicast reactivo).

- DSDV. (Destination-sequenced distance - vector protocol . Unicast proactivo).

- OLSR. COptimized link state routing 
protocol [3]. Unicast proactivo).

- LBM. (Location-based multicast. Geocast).

- GAMER. (Geocast adaptive mesh environment for routing. Geocast).

- GEOGRID. (Geocast).

- URAD (Unicast routing with area delivery) Geocast.

- GeoTORA (Geographic temporally ordered routing algorithm) Geocast.

Por las características particulares de las redes VANET es importante destacar un protocolo concebido para entornos vehiculares con densidad de tráfico no uniforme y donde no se puede arantizar la conectividad permanente, el cual usa la técnica de Store-carry-and-forward; ésta técnica se basa en una propuesta de Vhadat, llamada encaminamiento epidémico, el cual consiste o considera, que cuando un nodo está cerca de otro que se encuentra "infectado", refiriéndose a que tiene mensajes para propagar, este nodo o vehículo difunde el mensaje, cuando el nodo que no está infectado recibe el mensaje, se convierte en un nodo infectado con una probabilidad predeterminada, pero resulta difícil determinar dicha probabilidad de infección, pues la densidad y movilidad de los nodos es variable. De ahí la importancia de considerar muchos aspectos que pueden darse a fin de disminuir el margen de error, por ejemplo, dos vehículos que mantengan comunicación y de un momento a otro, uno de ellos se aleja, sin duda, la ruta definida anteriormente se pierde, el otro caso puede ser, cuando un carro que se encuentre en medio de dos vehículos infectados probablemente va a ser saturado con mensajes de ambos nodos.

A nivel de capa de transporte también se han definido algunas modificaciones o mejoras con el fin de ajustar TCP, para que se adapte a las particularidades de las redes MANET, dentro de estas mejoras de TCP encontramos:

- TCP-F. TCP feedback.
- $\quad$ TCP explicit.

- TCP-ELFN. Link failure notification

- TCPBuS. TCP buffering capability and sequence.

- TCP-Fixed-RTO. TCP fixed retransmission timeout.

- $\quad$ ATCP. Ad hoc TCP.

- TCP-DOOR. TCP detection of out of order and response.

Y el protocolo propuesto:

- ATP. Ad hoc transport protocol.

Para concluir lo relacionado con los conceptos de las redes VANET, vale la pena recordar que por tratarse de un sistema de red, también se corresponde con el modelo de red por capas como el OSI [19], donde en cada capa existe una serie de protocolos que van desde la capa de aplicación hasta la capa física, y que para el caso particular de este tipo de redes su medio de transmisión es inalámbrico [20] donde ya están definidos protocolos para éste nivel o capa.

\section{CADENAS DE MARKOV EN REDES VA- NET}

El concepto de cadenas de Markov es un tipo de proceso estocástico discreto en el que la probabilidad de que ocurra un evento depende del evento inmediatamente anterior; estas cadenas son utilizadas ampliamente ${ }^{7}$ en física, meteorología, finanzas, comunicaciones, motores de búsqueda como Google, en simulaciones para el análisis de problemas tales como el Modelo M/M/1. Para el caso de las redes MANET el movimiento individual de cada nodo o vehículo en redes VANET, es considerado en el análisis de patrón de movilidad, velocidad, dirección, cambio de longitud o tiempo entre dos puntos consecutivos y distribución de la posición de los móviles y donde cada modelo tiene sus propias métricas de movilidad.

La implementación y análisis de los mo-

7. http://es.wikipedia.org/wiki/Cadena_de_Markov 
delos de movilidad ha tenido muchos problemas con respecto a los escenarios reales [21], [22], en especial cuando se trata de implementar y medir o comparar los resultados de una simulación, en razón a que ponerlo en práctica conlleva a muchas limitaciones en especial porque en la actualidad, aun cuando se cuenta con avances significativos, es difícil encontrar vehículos con el hardware requerido para éste propósito [23], [24].

Un desplazamiento vehicular puede verse como una cadena de Markov cuyo espacio de estado esta dado por

$$
i=0, \pm 1, \pm 2, \ldots
$$

para un número

$$
\begin{gathered}
0<p<1 \\
r_{i, i+1}=p=r_{i, i-1}
\end{gathered}
$$

donde $\pi \mathrm{k}, \mathrm{l}$ representan la probabilidad de transición del estado k a l. se puede llamar paseo o desplazamiento aleatorio ${ }^{8}$ porque se ve en él como un modelo para un vehículo desplazándose en una línea recta que en cada momento del tiempo tiene la posibilidad ya sea de dar un giro hacia la derecha con probabilidad p, o dar el primer giro hacia la izquierda con probabilidad $1-p$, por lo tanto, puede notarse que el paseo o desplazamiento aleatorio es un proceso estocástico simple.

Un modelo probabilístico es la versión del desplazamiento aleatorio que utiliza cadenas de Markov para host en movimiento en redes ad-hoc conocido como MRW [25] (Markovian Random Walk), el cual define los siguientes tres estados:

- Estado 0, representa la posición actual del nodo móvil.

- Estado 1, representa la posición anterior del nodo móvil.

8. http://en.wikipedia.org/wiki/Random_walk
- Estado 2, representa la posición siguiente del nodo móvil.

La fig. 2 [26], [27]. muestra la cadena de Markov para el movimiento en las coordenadas X y Y de un nodo móvil.
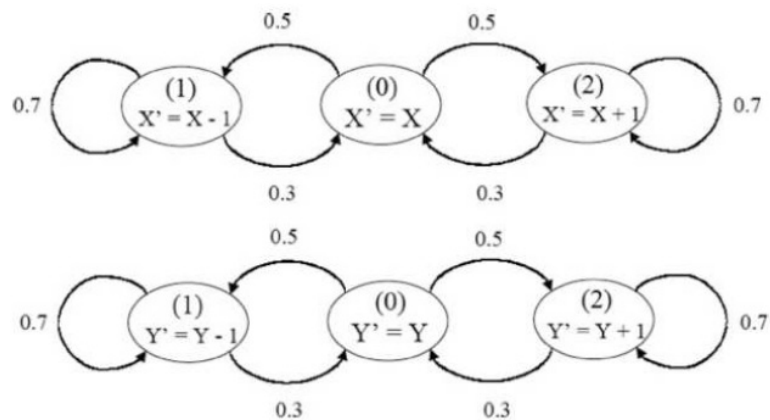

$$
\begin{array}{ll}
X^{\prime}: \text { next } x \text { coordinate } & X: \text { current } x \text { coordinate } \\
Y^{\prime}: \text { next } y \text { coordinate } & Y: \text { current } y \text { coordinate }
\end{array}
$$

Fig. 2. Cadena de markov y matriz de probabilidad.

Cada entrada de la matriz de probabilidad $P(i, j)$ representa la probabilidad de que el móvil pasará de un estado i al estado j por lo cual $\mathrm{P}(\mathrm{i}, \mathrm{j})$ se expresa como:

$$
P=\left\{\begin{array}{l}
P(0,0) P(0,1) P(0,2) \\
P(1,0) P(1,1) P(1,2) \\
P(2,0) P(2,1) P(2,2)
\end{array}\right\}
$$

Los valores dentro de esta matriz se utilizan para las actualizaciones de las posiciones X y Y del nodo móvil.

$$
P=\left\{\begin{array}{lll}
0.0 & 0.5 & 0.5 \\
0.3 & 0.7 & 0.0 \\
0.3 & 0.0 & 0.7
\end{array}\right\}
$$

Una vez que las probabilidades de transición de estados se definen, un nodo móvil puede dar un paso (estado) en cualquiera de las cuatro posibles direcciones: norte, sur, oriente u occidente, cabe señalar que los valores definidos prohíben movimientos entre la anterior y la siguiente posición, sin pasar a través de la ubicación actual. Una vez que un nodo móvil comien- 
za a moverse, es probable que conserve la misma dirección porque la probabilidad de que se mantenga en los estados 1 o 2 de la cadena de Markov es mayor que la probabilidad de que se regrese al estado 0 .

Este modelo probabilístico para el análisis de variables aleatorias es muy interesante y aplicable en toda su extensión a redes MANET en especial a las redes VANET, por lo cual un estudio más detallado se deja como propuesta para futuros trabajos de investigación.

\section{CONCLUSIONES}

Las redes MANET llevan alrededor de 20 años de investigación mientras que las VANET apenas 10 años, esto abre grandes oportunidades de trabajos de investigación que la academia debe motivar.

Gran parte de los temas que involucran las

Referencias Bibliográficas

[1] Popescu-Zeletin, R., I. Radusch, and M.A. Rigani, Vehicular-2-X Communication: State-of-the-Art and Research in Mobile Vehicular Ad hoc Networks. 2009: Springer Verlag.

[2] Baumann, R., S. Heimlicher, and M. May. Towards realistic mobility models for vehicular ad-hoc networks. 2007: IEEE.

[3] Moustafa, H. and Y. Zhang, Vehicular networks: techniques, standards, and applications. 2009: Auerbach $\mathrm{Pu}-$ blications Boston, MA, USA.

[4] Huang, C.M., C. Huang, and Y. Chang, Telematics communication technologies and vehicular networks: wireless architectures and applications. Information Science Reference-Imprint of: IGI Publishing Hershey, PA, 2009: p. 10-11.

[5] Skordylis, A. and N. Trigoni. Delay-bounded routing in vehicular ad-hoc networks. 2008: ACM. redes VANET se encuentran en fase de investigación, quizás tarde 10 o 20 años para ver los primeros resultados en forma masiva, lo cierto es que con la creciente industria automotriz y la exigencia por proteger el medio ambiente y vidas humanas, entre otras razones, hay una fuerte presión para llevar a las redes VANET a convertirse no solo en un asunto interesante sino también una exigencia.

Las cadenas de Markov se convierten en una herramienta muy importante para el análisis de redes espontáneas vehiculares o simplemente VANET, en especial en lo que hace referencia a los protocolos de encaminamiento, toda vez que las características de estas son muy particulares lo cual lleva a desarrollos no solo desde el punto de vista de simulación sino matemático.

[6] Hartenstein, H., K. Laberteaux, and E. Corporation, VANET Vehicular Applications and Inter-Networking Technologies. 2010: Wiley Online Library.

[7] Xiong, N., et al., A Resilient and Scalable Flocking Scheme in Autonomous Vehicular Networks. Mobile Networks and Applications, 2010. 15(1): p. 126-136.

[8] Gabarrón, J.B.T., E.E. López, and J.G. Haro, Evaluacion de mecanismos de priorizacion en $802.11 \mathrm{p}$ con VHDL.

[9] Sarkar, N.I., The Impact of Transmission Overheads on IEEE 802.11 Throughput: Analysis and Simulation. 2011.

[10] Efatmaneshnik, M., A.T. Balaei, and A. Dempster. A Channel Capacity Perspective on Cooperative Positioning Algorithms for VANET: Citeseer. 
[11] Chen, Y.S., Y.W. Lin, and S.L. Lee, A mobicast routing protocol in vehicular ad-hoc networks. Mobile Networks and Applications, 2010. 15(1): p. 20-35.

[12] Khan, I., PERFORMANCEEVALUATION OF AD HOC ROUTING PROTOCOLS FOR VEHICULAR AD HOC NETWORKS. 2009.

[13] Tripp Barba, C., K. Ornelas Aguirre, and M. Aguilar Igartua. Performance evaluation of a hybrid sensor and vehicular network to improve road safety. 2010: ACM.

[14] Schoch, E., et al., Communication patterns in vanets. Communications Magazine, IEEE, 2008. 46(11): p. 119-125.

[15] Lee, K.C., U. Lee, and M. Gerla, Survey of Routing Protocols in Vehicular Ad Hoc Networks. Advances in Vehicular Ad-Hoc Networks: Developments and Challenges, IGI Global, 2009.

[16] Joshi, H.P., M.L. Sichitiu, and M. Kihl, Distributed Robust Geocast.
[17] Bakhouya, M., J. Gaber, and M. Wack. Performance evaluation of DREAM protocol for inter-vehicle communication: IEEE.

[18] Banik, A.K., Routing Protocol with prediction based mobility model in Vehicular Ad Hoc Network (VANET). 2010.

[19] Martinez, F.J., et al., A survey and comparative study of simulators for vehicular ad hoc networks (VANETs). Wireless Communications and Mobile Computing, 2009.

[20] Baldessari, R., A. Festag, and J. Abeillé. Nemo meets vanet: a deployability analysis of network mobility in vehicular communication. 2007: IEEE.

[21] Shirani, R. and F. Hendessi. A Markov chain model for evaluating performance of Store-Carry-Forward procedure in VANETs: IEEE.

[22] Yang, L.T., et al., A markov-based packet-level error model for VANET and predicting. Dianzi Xuebao(Acta Electronica Sinica), 2009. 37(10). 